\title{
Finite Element Stereo Digital Image Correlation: framework and mechanical regularisation
}

\author{
Jean-Emmanuel Pierré • Jean-Charles \\ Passieux · Jean-Noël Périé
}

Received: date / Accepted: date

\begin{abstract}
The use of Finite Element meshes in Digital Image Correlation (FEDIC) is now widespread in experimental mechanics. Up to now FE have been much less used in Stereo-DIC. The first goal of this paper is to explain in details how to use FE in Stereo-DIC using a formulation in the physical coordinate system. More precisely, it is shown how to perform the calibration of possibly nonlinear models, shape and displacement measurement based on a FE mesh. In addition it is shown that with such a framework it is possible to regularise the measurement with a FE model based on the same mesh. For instance, using this technique, it is shown that it is possible to measure the rotation field of a bending plate in addition to its displacement.
\end{abstract}

Keywords Digital Image Correlation · Stereovision · Finite Element · Mechanical Regularisation · Plate kinematics

J.-E. Pierré, J.-C. Passieux ${ }^{\star}$, J.-N. Périé

Université Fédérale Toulouse Midi-Pyrénées - Institut Clément Ader - CNRS UMR 5312 INSA/UPS/Mines Albi/ISAE - 3 rue Caroline Aigle 31400 Toulouse - France

^Corresponding author:

Tel.: +33-(0)561171179

E-mail: passieux@insa-toulouse.fr 


\section{Introduction}

Digital Image Correlation $(D I C)$ and Digital Volume Correlation $(D V C)$, 6, 11 , 24 are now widely used in experimental mechanics. This family of methods aims at measuring $2 \mathrm{D}$ or $3 \mathrm{D}$ displacement fields from digital planar or volume images, respectively. In their initial version, the idea was to search for the best parameters of a given transformation to register small independent subsets of pixels. In the context of experimental mechanics, the use of Finite Elements in the context of DIC/DVC (FE-DIC/DVC [23,2]) has been extensively developed during the last decade. Among many advantages, to us, the main interest relies on the fact that it provides a very simple bridge between experiments and simulations, which is very convenient for validation [16], identification purposes [9, 19, 14] and for performing mechanically regularised measurements.

Even in situations where 2D-DIC could be used, Stereo-DIC is preferred because of both accuracy and simplicity of the setup 5, 25. Classically, the idea of Stereo-DIC is to use one stereoscopic image pair $f_{1}$ and $f_{2}$ in a reference configuration at $t_{0}$ and a second one $g_{1}$ and $g_{2}$ at $t_{0}+d t$ in a deformed configuration, see Fig. 1

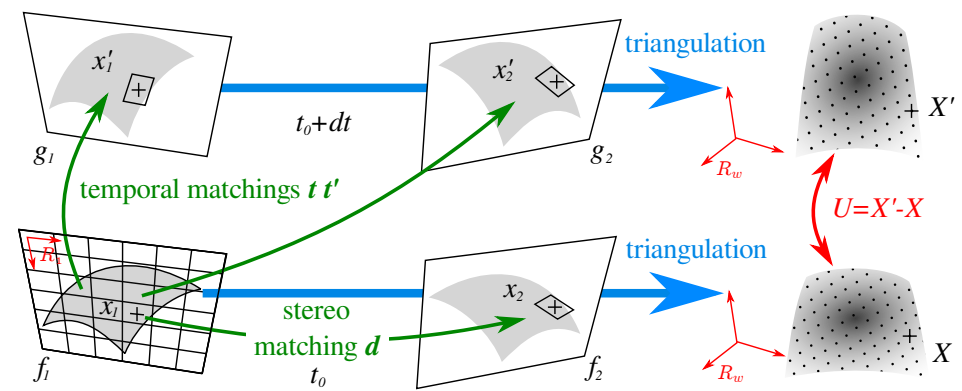

Fig. 1 Main steps of a 3D displacement field measurement of a surface with classical StereoDigital Image Correlation. Pairings are in green and triangulations in blue

Classical Stereo-DIC procedure involves a chain of optimisation problems: 


\begin{tabular}{ll}
\hline Calibration: & 1) find ex/in-trinsic parameters from a set of images of calibration grids \\
\hline Shape Measurement: & 2) find matching field $\mathbf{d}$ between reference images $f_{1}$ and $f_{2}$ \\
& 3) triangulation: find $\mathbf{X}$ at $t_{0}$ from $\mathbf{x}_{1}$ and $\mathbf{x}_{2}=\mathbf{x}_{1}+\mathbf{d}\left(\mathbf{x}_{1}\right)$ \\
\hline Displacement Measurement: & 4) find matching $\mathbf{t}$ between images $f_{1}$ and $g_{1}$ \\
& 5) find matching $\mathbf{t}^{\prime}$ between images $f_{1}$ and $g_{2}$ \\
6) triangulation: find $\mathbf{X}$ at $t_{0}+d t$ from $\mathbf{x}_{1}^{\prime}=\mathbf{x}_{1}+\mathbf{t}\left(\mathbf{x}_{1}\right)$ and $\mathbf{x}_{2}^{\prime}=\mathbf{x}_{1}+\mathbf{t}^{\prime}\left(\mathbf{x}_{1}\right)$
\end{tabular}

Eventually, the 3D displacement is estimated by comparing measured shapes: $\mathbf{U}=\mathbf{X}^{\prime}-\mathbf{X}$. The drawback of this classical Stereo-DIC formulation is that the displacement is not the unknown of a unique optimization problem as it is the case with 2D-DIC and DVC. In this situation, the bridge between measurement and simulation is less direct. In particular, applying a mechanical regularisation is not possible in this framework.

In opposition to DIC and DVC, the literature on FE-Stereo-DIC (or FE-SDIC) is much less prolific certainly because the extension is not straightforward. The first attempt was proposed in 22 , where a theoretical FE mesh was used to perform the displacement measurement solution of a unique problem defined in the world coordinate system. In this paper this formulation is not fully exploited since it is not used for calibration and shape measurement. A very similar global stereo formulation based on IsoGeometric Analysis $(I G A)$ was proposed in [1, 3]. It is used not only for the displacement measurement, but also for calibration of a linear camera model and shape measurement.

In this paper, we develop and detail a general framework covering all the aspects of FE-Stereo-DIC. It consists in a formulation in the physical coordinate system which makes possible to calibrate a stereo rig (with possibly non-linear camera models), to measure the actual shape of the specimen and to measure the displacement field, using a FE interpolation. A technique is proposed to build the minimal number of integration points in this context. In addition, it become possible to add a regularisation based on a FE mechanical model. For instance, the method is illustrated with a measurement of the kinematic fields of a bending 
plate; the kinematic fields meaning in this case not only displacements but also rotations.

In Sect. 2 the three problems of calibration, shape measurement and displacement measurement will be rewritten as three optimisation problems where the unknowns are the intrinsic/extrinsic parameters, the shape and 3D displacement respectively. Then, in Sect. 3, it will be shown that, with the proposed framework, it is directly possible to add a constraint of regularity such as a mechanical regularisation term. It will be also shown that the use of a plate model in the regularisation, is likely to provide accurate rotation fields. Finally in Sect. 4, both synthetic and real test case will be presented in order to illustrate the efficiency of the method, the effects of the parameters and its robustness with respect to noise.

\section{Rewriting Stereo-DIC problem in the world reference system with}

\section{Finite Elements}

This section contains the three parts of the measurement of a $3 \mathrm{D}$ displacement in the reference system $R_{W}$ described by the mesh. Indeed, with an FE mesh, the theoretical shape does not necessarily correspond to the real one. Therefore, a displacement measurement needs two preliminary steps (Fig. 2). Firstly, cameras

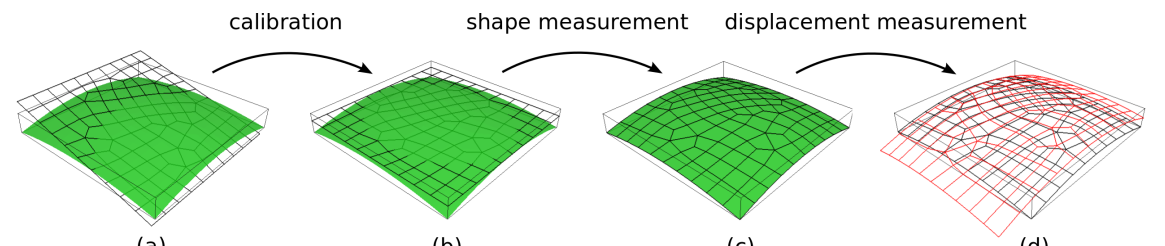

(a)

(b)

(c)

(d)

Fig. 2 Steps of the new Stereo-DIC formulation with a Finite-Element mesh (FE-SDIC): (a) first guess of the theoretical CAD mesh plate (black) on the real (a priori unknown) plate (green) before any calibration or shape measurement. (b) Calibration of the extrinsic parameters by DIC (with fixed 3D points $\mathbf{X}$ ). (c) Shape measurement (with fixed parameters $\mathbf{p}_{c}$ ). (d) Temporal measure of the 3D displacement $\mathbf{U}$ (from the black position of the mesh to the red one)

have to be located in the world reference system. This is called the extrinsic parameters calibration but intrinsic parameters can also be optimised ac- 
cording to the used mesh Sect. 2.1. Then, the mesh shape has to be corrected: the projection of each points of the mesh in both cameras (with their projectors $\mathbf{P}_{c}$ ) have to be stereo-matching (the corresponding gray-levels must be equals). This step is the shape measurement Sect. 2.2. A very similar approach has already been published in [1] for a CAD-based shape measurement with NURBS. Finally, the 3D displacement between two deformation steps can be measured Sect. 2.3 .

2.1 Extrinsic and Intrinsic parameters calibration

In this study, the method being based on a Gauss Newton algorithm, the calibration has to be initialised with the Stereo Software Vic- $3 \mathrm{D}^{\mathrm{TM}}$. The calibration consists in finding all parameters $\mathbf{p}_{c}$ of each camera's projector $\mathbf{P}_{c}$ :

-6 extrinsic parameters in order to find the position of the camera's reference system in $R_{W}: 3$ translations and 3 rotations.

- 4 linear intrinsic parameters in order to project a point from the camera to its image : 2 focal lengths $\left(f_{u}, f_{v}\right)$ and two centers of image $\left(c_{u}, c_{v}\right)$ along both horizontal $u$ and vertical $v$ directions.

- at least 2 non-linear intrinsic parameters : the first order of radial distortion $\kappa$ and the skew angle.

More details on the non-linear models of cameras can be found in [5],17]. In this paper, the intrinsic parameters are initialised with $\mathrm{Vic}-3 \mathrm{D}^{\mathrm{TM}}$. The first problem consists in finding the extrinsic parameters in order to locate the 3D Finite Element mesh (considered rigid) as close as possible of the real surface which is unknown. In the following, $\mathbf{p}_{c}$ denote the extrinsic parameters of the projector $\mathbf{P}_{c}$ associated to the image $f_{c}$ (the intrinsic ones are fixed). For the sake of simplicity, we first consider a standard stereo bench, i.e. $c=\{1 ; 2\}$. The parameters $\mathbf{p}_{c}$ are assessed by minimizing the following objective functional based on the gray-level conservation assumption: 


$$
\mathbf{p}_{1}^{\star}, \mathbf{p}_{2}^{\star}=\underset{\mathbf{p}_{1}, \mathbf{p}_{2}}{\arg \min } \int_{\Omega}\left[f_{1}\left(\mathbf{P}_{1}\left(\mathbf{X}, \mathbf{p}_{1}\right)\right)-f_{2}\left(\mathbf{P}_{2}\left(\mathbf{X}, \mathbf{p}_{2}\right)\right)\right]^{2} d \mathbf{X}
$$

where $\mathbf{X}$ stands for a $3 \mathrm{D}$ point located on the visible surface $\Omega$ of the specimen.

As said previously, the quadrature is done in the $3 \mathrm{D}$ domain and an image is not preferred over another (there is no concept of master-slave), each camera being treated symmetrically. This non-linear problem is solved with a Newton algorithm. At iteration $k$, parameters $\mathbf{p}=\left[\mathbf{p}_{1}, \mathbf{p}_{2}\right]^{T}$ are searched in the form $\mathbf{p}^{k+1}=\mathbf{p}^{k}+\delta \mathbf{p}$. After linearisation and differentiation, the problem reads:

$$
\begin{gathered}
\mathbf{M}_{\text {ext }}^{k} \delta \mathbf{p}=\mathbf{b}_{\text {ext }}^{k} \\
\left\{\begin{array}{c}
M_{e x t, i j}^{k}=\int_{\Omega}\left(\frac{\partial \mathbf{P}_{1}}{\partial p_{i}} \nabla f_{1}-\frac{\partial \mathbf{P}_{2}}{\partial p_{i}} \nabla f_{2}\right)\left(\frac{\partial \mathbf{P}_{1}}{\partial p_{j}} \nabla f_{1}-\frac{\partial \mathbf{P}_{2}}{\partial p_{j}} \nabla f_{2}\right)^{T} d \mathbf{X} \\
\mathbf{b}_{e x t, i}^{k}=-\int_{\Omega}\left(\frac{\partial \mathbf{P}_{1}}{\partial p_{i}} \nabla f_{1}-\frac{\partial \mathbf{P}_{2}}{\partial p_{i}} \nabla f_{2}\right)\left(f_{1}\left(\mathbf{P}_{1}\left(\mathbf{X}, \mathbf{p}_{1}^{k}\right)\right)-f_{2}\left(\mathbf{P}_{2}\left(\mathbf{X}, \mathbf{p}_{2}^{k}\right)\right)\right) d \mathbf{X}
\end{array}\right.
\end{gathered}
$$

where $\frac{\partial \mathbf{P}_{c}}{\partial p_{i}}$ are the projectors $\mathbf{P}_{c}$ gradient with respect to the extrinsic parameters (even with a non-linear model, an analytical expression is known). $\nabla f_{c}$ is image $f_{c}$ gradient at $\mathbf{P}_{c}\left(\mathbf{X}, \mathbf{p}_{c}^{k}\right)$ which depends of $\mathbf{p}_{c}$. This means that the operator $\mathbf{M}_{\text {ext }}^{k}$ has to be re-assembled at each iteration.

For a Gradient algorithm, the initial guess should be close to the solution. The first guess can be obtained "by hand" by selecting some points in each image and on the mesh (it would also be possible to detect at least three particular points with a pattern recognition algorithm). Then, a small non-linear problem has to be solved. This problem has the same expression than the bundle adjustment (2) 28, but here, $\mathbf{X}, \mathbf{x}_{1}, \mathbf{x}_{2}=\mathbf{x}_{1}+\mathbf{d}\left(\mathbf{x}_{1}\right)$ are fixed and only the extrinsic parameters are searched for:

$$
\mathbf{p}_{1}^{\star}, \mathbf{p}_{2}^{\star}=\underset{\mathbf{p}_{1}, \mathbf{p}_{2}}{\arg \min }\left\|\mathbf{P}_{1}\left(\mathbf{X}, \mathbf{p}_{1}\right)-\mathbf{x}_{1}\right\|_{2}^{2}+\left\|\mathbf{P}_{2}\left(\mathbf{X}, \mathbf{p}_{2}\right)-\mathbf{x}_{1}-\mathbf{d}\left(\mathbf{x}_{1}\right)\right\|_{2}^{2}
$$

In order to illustrate these steps, on Fig. 2(a) can be seen the first guess of the position of the mesh on the real surface. Fig. 2(b) is after the extrinsic calibration 
(eq. (1)). In these two steps, the mesh $\mathbf{X}$ is considered fixed in its reference system and the goal is to position this system as close as possible to the unknown surface by finding the rigid translations and rotations.

In paper [1], some intrinsic parameters of linear camera models are estimated using the same framework (using the speckle pattern of the specimen only). But the measurement of a least one distance is needed to get an absolute estimate of, for instance, the focal length. Here, the model of camera is more complex (non-linear with distortions) and the initial mesh is flat. Thus, intrinsic parameters cannot be optimised at the same time than the extrinsic ones. The idea would be to release all intrinsic parameters once the extrinsic are optimized. The functional is the same (eq. (1)) but with $\mathbf{p}_{c}$ containing only intrinsic parameters $\left(c_{u}, c_{v}, f_{u}, f_{v}, \kappa, s k e w\right)$ (the extrinsic ones are now fixed). It is possible to alternate these optimizations.

In practice, and in order to be more generic, we decided to calibrate the intrinsic parameters using classic planar calibration targets. This step was done using Vic$3 \mathrm{D}^{\mathrm{TM}}$. It was verified that these parameters were very close to the local minimum of the above graylevel based functional.

\subsection{Shape measurement with an FE mesh}

Once all cameras' parameters $\mathbf{p}_{c}$ are known (and optimised), the shape has to be corrected. Measuring the shape means finding the position $\mathbf{X}$ verifying the graylevel conservation. Here, the latter is verified between the reference images of each camera. The same method as for the cameras' parameter is used but the unknowns are the $3 \mathrm{D}$ positions $\mathbf{X}$ :

$$
\mathbf{X}^{\star}=\underset{\mathbf{X}}{\arg \min } \int_{\Omega}\left[f_{1}\left(\mathbf{P}_{1}\left(\mathbf{X}, \mathbf{p}_{1}\right)\right)-f_{2}\left(\mathbf{P}_{2}\left(\mathbf{X}, \mathbf{p}_{2}\right)\right)\right]^{2} d \mathbf{X}
$$

At iteration $k$, the estimation of the position is written $\mathbf{X}^{k+1}=\mathbf{X}^{k}+\delta \mathbf{X}$ where the displacement correction $\delta \mathbf{X}$ is searched in the finite element subspace with 
$\delta \mathbf{X}=\sum_{i} \mathbf{N}_{i}(\mathbf{X}) q_{i}:$

$$
\begin{gathered}
\mathbf{M}_{\text {shape }}^{k} \mathbf{q}=\mathbf{b}_{\text {shape }}^{k} \\
\left\{\begin{array}{c}
\mathbf{M}_{\text {shape }, i j}^{k}=\int_{\Omega} \mathbf{N}_{i}^{T}\left(\nabla \mathbf{P}_{1} \nabla f_{1}-\nabla \mathbf{P}_{2} \nabla f_{2}\right)\left(\nabla \mathbf{P}_{1} \nabla f_{1}-\nabla \mathbf{P}_{2} \nabla f_{2}\right)^{T} \mathbf{N}_{j} d \mathbf{X} \\
\mathbf{b}_{\text {shape }, i}^{k}=-\int_{\Omega} \mathbf{N}_{i}^{T}\left(\nabla \mathbf{P}_{1} \nabla f_{1}-\nabla \mathbf{P}_{2} \nabla f_{2}\right)\left(f_{1}\left(\mathbf{P}_{1}\left(\mathbf{X}^{k}, \mathbf{p}_{1}\right)\right)-f_{2}\left(\mathbf{P}_{2}\left(\mathbf{X}^{k}, \mathbf{p}_{2}\right)\right)\right) d \mathbf{X}
\end{array}\right.
\end{gathered}
$$

where $\nabla \mathbf{P}_{c}$ are the projectors' spatial gradient which have an analytical form. As for the calibration, the operator $\mathbf{M}_{\text {shape }}^{k}$ has to be re-assemble at each iteration. After convergence, an estimation of the real shape is obtained (see Fig. 2(c)).

Remarks:

- Since this is based on a Gauss Newton, this algorithm requires an initialisation step. Usually, a multigrid initialisation with a pixel aggregation (coarse graining) is used [18. But unlike what is usually done in 2D-DIC, where this step is made with a coarse mesh, here the same mesh is used with a decreasing Tikhonov regularisation in order to avoid 3D projection of fields from a mesh to another.

- As said previously, $\mathbf{M}_{\text {shape }}^{k}$ has to be re-assemble at each iteration. This has a significant cost because here, the reference images of all cameras are compared by pairs. For $n$ cameras, there are $\frac{n(n-1)}{2}$ couple of cameras. On the other hand, in [4, a reference object $\hat{f}$ with an intrinsic texture is created. Thus, all images are compared to $\hat{f}$ which is faster when using four or more cameras because only $n$ couples are considered.

Regularisation As always in DIC, this problem is ill-posed. With the classical approach, there are two unknowns (the components of the left-right disparity field d on the image) for only one scalar equation (gray-level conservation). Here, a 3D field is searched for, which means that for the same number of equation, there is one more unknown. Indeed, in the problem 3 the mesh can slide along the surface of the object. There can be a global sliding (see Fig. 3(b) which imply that the dimension of the operator's kernel is at least 3 (two sliding along two surface directions and one rotation). But in the formulation, the problem is even 
harder because each node can moves relatively to another (see Fig. 3(c) without changing the value of the functional. Thus, a higher degree of regularisation has to be added to the functional.

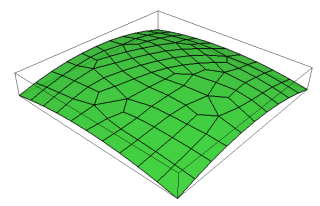

(a)

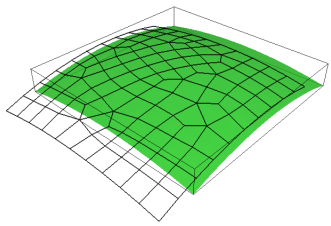

(b)

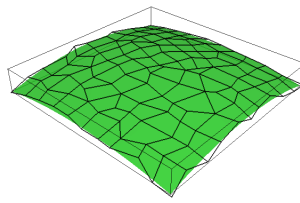

(c)

Fig. 3 Illustration of the ill-posed problem of shape measurement. (a) Theoretical position of the mesh after calibration and shape measurement. (b) Global or (c) local sliding during the shape measurement

For the local sliding, an isometric constraint can be imposed, like a Tikhonov regularisation term:

$$
\delta \mathbf{X}^{\star}=\underset{\delta \mathbf{X}}{\arg \min } \int_{\Omega}\left[f_{1}\left(\mathbf{P}_{1}\left(\mathbf{X}+\delta \mathbf{X}, \mathbf{p}_{1}\right)\right)-f_{2}\left(\mathbf{P}_{2}\left(\mathbf{X}+\delta \mathbf{X}, \mathbf{p}_{2}\right)\right)\right]^{2} d \mathbf{X}+\lambda\|\delta \mathbf{X}\|_{\mathbf{K}}^{2}
$$

where the norm $\|\cdot\|_{\mathbf{K}}$ can be associated to a mechanical operator $\mathbf{K}$ (truss, stiffness plate/shell or Laplace). One of the interest of using SDIC with NURBS [1], rely on the fact that only a few control points are necessary to describe a surface which indirectly regularise the problem. Indeed, the shape interpolation can be uncoupled from the displacement interpolation which can be refined without modifying the shape. In FE, this uncoupling is not possible and the same mesh is used for both shape and displacement measurement. Thus, it must be regularised externally.

However, both NURBS and FE approaches are subject to global sliding. The previous regularisation does not prevent that problem. These shifts are particularly visible when iterating between the optimisation of the cameras' parameters and the shape measurement.

To avoid these shifts and restore the uniqueness of the problem, it has been decided to seek the correction of the nodal displacement along the normal to the 
surface [13. The problem has then only one dof per node. In practice, the surface normal is estimated at each node (average of the neighbouring element normals) in the initial shape of the mesh.

\section{$2.33 \mathrm{D}$ displacement measurement}

Once the real shape (thus, the real mesh) is known, it is possible to measure the 3D displacement $\mathbf{U}$ between two steps $\left(t_{0}\right.$ and $\left.t_{0}+d t\right)$. Unlike Classical Stereo-DIC, the three optimisation problems are turned into on single problem where $\mathbf{U}$ is the unique unknown. The main idea for this reformulation is to work only in the 3D mesh, chosen to be the world reference system $R_{W}$. All problems are written in this $3 \mathrm{D}$ coordinate system and weak-form integrals of the gray-level conservation are also in $R_{W}$.

For each 3D point $\mathbf{X}$, the projector $\mathbf{P}_{c}$ gives a point $\mathbf{x}$ in the image. Obviously, it is also possible to have the projection of the displaced point $\mathbf{X}^{\prime}=\mathbf{X}+\mathbf{U}(\mathbf{X})$ (at $\left.t_{0}+d t\right)$ in the deformed state images $g_{c}$ (see Fig. 4). The point $\mathbf{X}$ is projected on the reference image $f_{c}$ and $\mathbf{X}^{\prime}$ is projected on the deformed state image $g_{c}$. Their gray level should be equals and this, for each camera $c$. Thus, the functional reads:

$$
\mathbf{U}^{\star}=\underset{\mathbf{U}}{\arg \min } \sum_{c} \int_{\Omega}\left[f_{c}\left(\mathbf{P}_{c}\left(\mathbf{X}, \mathbf{p}_{c}\right)\right)-g_{c}\left(\mathbf{P}_{c}\left(\mathbf{X}+\mathbf{U}(\mathbf{X}), \mathbf{p}_{c}\right)\right)\right]^{2} d \mathbf{X}
$$

which is just the gray level conservation equation for each camera. This correspond to the vertical pairing in Fig. 4. Each term of each camera being independent, it is possible to consider more than two cameras [4]. Such a formulation in the world reference system was first introduced in [22]. The benefit is to symmetrically use each camera. At iteration $k$, a correction of the 3D displacement field is introduced $\mathbf{U}^{k+1}=\mathbf{U}^{\mathrm{k}}+\sum_{i} \mathbf{N}_{i}(\mathbf{X}) q_{i}$ and the problem reads:

$$
\underbrace{\left(\sum_{c} \mathbf{M}_{d i c}^{c, k}\right)}_{\mathbf{M}_{\text {stereo }}^{k}} \mathbf{q}=\underbrace{\left(\sum_{c} \mathbf{b}_{d i c}^{c, k}\right)}_{\mathbf{b}_{\text {stereo }}^{k}}
$$




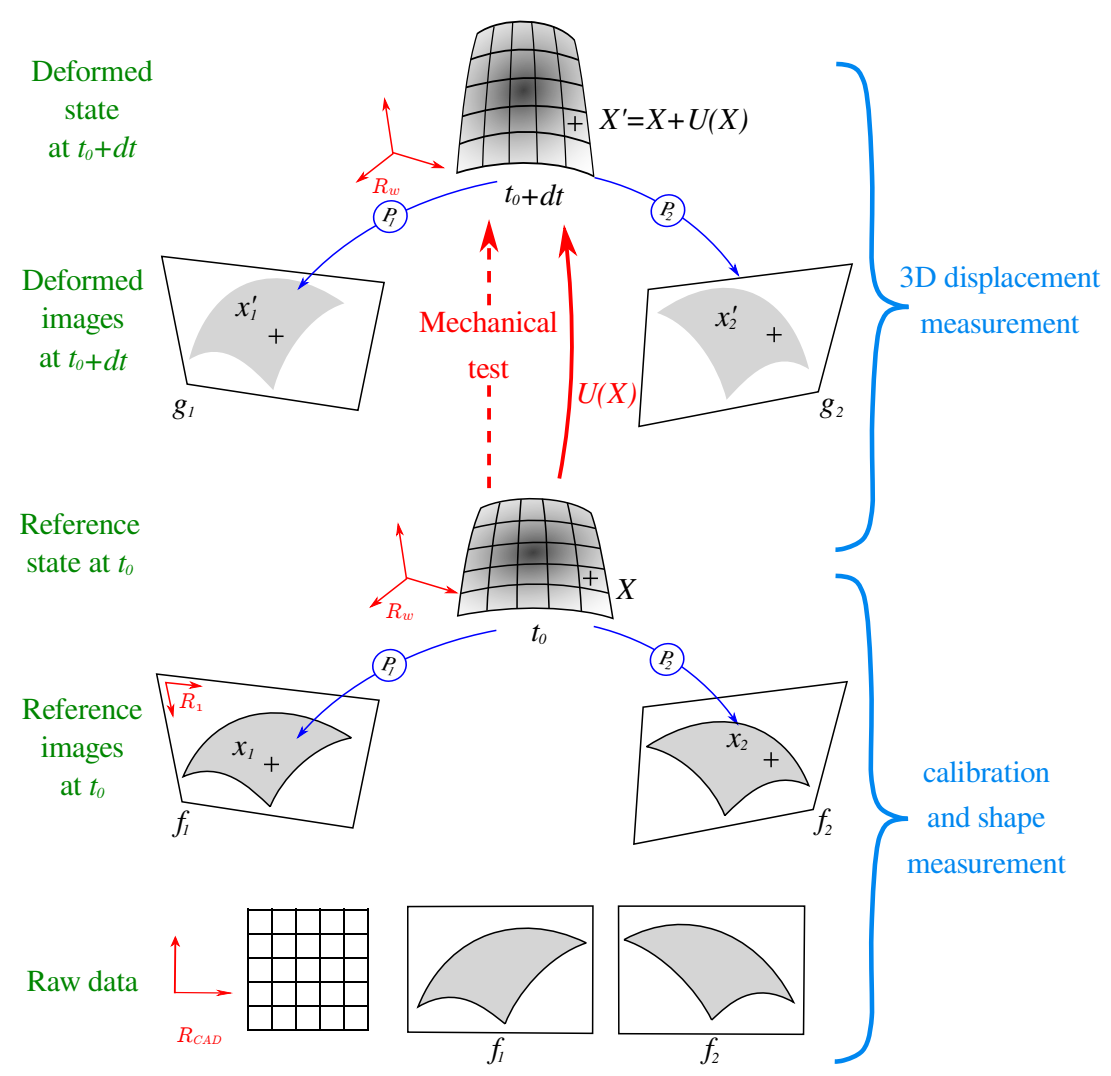

Fig. 4 3D displacement measurement in Stereo-DIC with a finite element based method (FESDIC)

$$
\left\{\begin{aligned}
\mathbf{M}_{d i c, i j}^{c, k} & =\int_{\Omega} \mathbf{N}_{i}^{T}\left(\nabla \mathbf{P}_{c} \nabla f_{c}\right)\left(\nabla \mathbf{P}_{c} \nabla f_{c}\right)^{T} \mathbf{N}_{j} d \mathbf{X} \\
\mathbf{b}_{d i c, i}^{c, k} & =\int_{\Omega} \mathbf{N}_{i}^{T}\left(\nabla \mathbf{P}_{c} \nabla f_{c}\right)\left(f_{c}\left(\mathbf{P}_{c}\left(\mathbf{X}, \mathbf{p}_{c}\right)\right)-g_{c}\left(\mathbf{P}_{c}\left(\mathbf{X}+\mathbf{U}^{k}, \mathbf{p}_{c}\right)\right)\right) d \mathbf{X}
\end{aligned}\right.
$$

Remarks:

- Both left and right hand side are sums of independent operator on each camera.

This implies that this problem can be parallelized like what is done in domain decomposition method in 2D-DIC [15].

- Like shape measurement, this algorithm needs an initialisation step such as a multigrid initialisation based on a pixel aggregation (coarse graining) and a Tikhonov regularisation with a Laplacian operator. 
- The correlation operator $\mathbf{M}_{\text {stereo depends on the displacement (because of }}^{k}$ the gradient of the projectors $\left.\nabla \mathbf{P}_{c}\left(\mathbf{X}+\mathbf{U}^{k}\right)\right)$ which means once again that it should be re-assemble at each iteration. In practice, this is not done because the multigrid initialisation is close to the actual displacement. Thus, no significant difference between re-assembling or not was found (except that it is far more time consuming)

To avoid a time drift of the pairing, it is also possible to introduce an additional term which minimize the difference of gray level between the deformed state images at $t_{0}+d t$ taken in pairs (this corresponds to an horizontal pairing in Fig. 4):

$$
\begin{gathered}
\mathbf{U}^{\star}=\underset{\mathbf{U}}{\arg \min } \sum_{c} \int_{\Omega}\left[f_{c}\left(\mathbf{P}_{c}(\mathbf{X}), \mathbf{p}_{c}\right)-g_{c}\left(\mathbf{P}_{c}\left(\mathbf{X}+\mathbf{U}(\mathbf{X}), \mathbf{p}_{c}\right)\right)\right]^{2} d \mathbf{X} \\
+\sum_{c} \sum_{e<c} \int_{\Omega}\left[g_{c}\left(\mathbf{P}_{c}\left(\mathbf{X}+\mathbf{U}(\mathbf{X}), \mathbf{p}_{c}\right)\right)-g_{e}\left(\mathbf{P}_{e}\left(\mathbf{X}+\mathbf{U}(\mathbf{X}), \mathbf{p}_{e}\right)\right)\right]^{2} d \mathbf{X}
\end{gathered}
$$

the pairing at $t_{0}$ is already verified with the calibration and the shape measurement. Of course, with this last term, a non-negligible cost is added when more than two cameras are used.

Quadrature. In all previous equations, quadrature is done in the 3D world reference system and not on the images as with classical DIC. A dedicated quadrature method has to be set up. Contrarily to simulation where a Gauss method is efficient, here, the integrand involves the grayscale gradient which has a very short variation length. Thus a Riemann integral method was therefore chosen by splitting homogeneously and homothetically each finite element. This choice was proven to be more accurate [17]. In practice, a smaller number of points can be build with an equivalent accuracy. It considers an inhomogeneous partition of quadrangles and triangles, in the isoparametric coordinate system, build from homogeneous partition of the two smallest edges. On Fig. 5, we show that such a technique reduces significantly the number of integration points. According to our tests, using this method, their are rarely more than one integration point per pixel. 


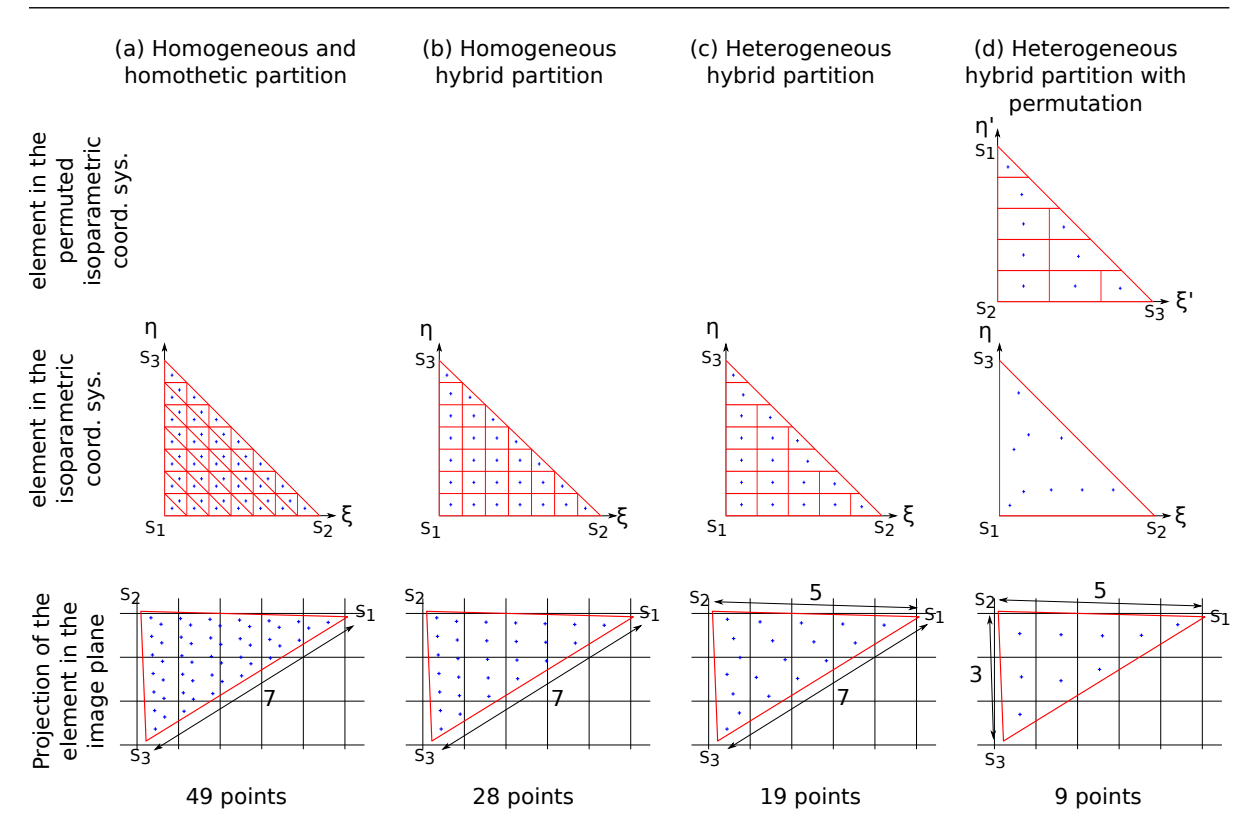

Fig. 5 Example of a different quadrature rule in the isoparametric coordinate system in order to find as few integration points as possible

Remark. In this article, this formulation in the 3D coordinate system is needed in order to add a mechanical regularisation term to the Stereo-DIC measure. But this is also interesting in 2D-DIC [17] for two reasons:

- The pixel based quadrature does not integrate accurately a constant function.

The area of an element is approximated by an integer number of pixels and depends on the point of view of the camera. Even the shape of the elements are not accurate because of the linear approximation of the element edges: with distortions the projection of a segment is not necessarily linear. This has a weak impact on the standard uncertainty of the measure but can improve the systematic bias of an order of magnitude even with a linear simple model of camera.

- A more complex model of camera with distortions can be used which drastically decreases measurement uncertainties. This is usefull when identifying parameters with a FEMU method based on displacement (FEMU-U). It is also 
an essential method for crack propagation based on Williams series if it is not possible to use a telecentric lens.

\section{Stereo-DIC and Plate Regularisation (R-FE-SDIC)}

Once having the 3D displacement as the unique unknown, it is easier to add a regularisation term. The idea is not only to make a measurement, but also to filter the latter with a mechanical model. This is very important when trying to measure accurate rotation fields.

Furthermore, for a plate or shell model, not only displacements but also rotations at the mid-surface are considered (see Fig. 6). The 3D displacement $\mathbf{U}$ measured with Stereo-DIC is the displacement of the observed surface which is the top one. Here, we propose a method in order to measure directly the midplane unknowns.

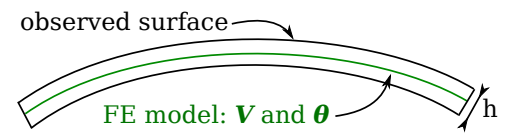

Fig. 6 Representation of a 3D plate or shell of a thickness $h$. The 3D displacement U measured with an optical bench is on the observed surface whereas the plate or shell model considers displacements $\mathbf{V}$ and rotations $\theta$ at the mid-surface

\subsection{Plate kinematic Regularisation}

With a plate theory (such as the Mindlin-Reissner theory of plates), a simple linear projector $\Pi$ can be used to determine the displacement of the upper skin depending upon the displacements $\mathbf{V}$ and rotations $\theta$ of the mid-surface. By extension and for a sake of simplicity, the vector of unknowns containing displacements and rotations 
of the mid-surface is denoted by $\mathbf{V}$.

$$
\mathbf{U}=\Pi \mathbf{V} \Leftrightarrow\left[\begin{array}{c}
U_{x} \\
U_{y} \\
U_{z}
\end{array}\right]=\Pi\left[\begin{array}{c}
V_{x} \\
V_{y} \\
V_{z} \\
\theta_{x} \\
\theta_{y}
\end{array}\right]
$$

Then the Stereo-DIC problem can be written:

$$
\begin{aligned}
\mathbf{V}^{\star}=\underset{\mathbf{V}}{\arg \min } & \frac{1-\lambda_{m}}{\alpha_{v}} \sum_{c} \int_{\Omega}\left[f_{c}\left(\mathbf{P}_{c}\left(\mathbf{X}, \mathbf{p}_{c}\right)\right)-g_{c}\left(\mathbf{P}_{c}\left(\mathbf{X}+\Pi \mathbf{V}(\mathbf{X}), \mathbf{p}_{c}\right)\right)\right]^{2} d \mathbf{X} \\
& +\frac{\lambda_{m}}{\alpha_{m}}\|\overline{\mathbf{K}} \mathbf{V}\|_{2}^{2}
\end{aligned}
$$

where the unknown is not only the displacement of the upper skin $\mathbf{U}$, but the five degrees of freedom $\mathbf{V}$ of the mid-surface. $\alpha_{v}$ and $\alpha_{m}$ are coefficients for the weighting of the terms of vision and stiffness. Thus, $\lambda_{m}$ is a dimensionless coefficient for the mechanical regularisation term set between 0 and 1 (see Sect. 4.1.3). Finally, $\overline{\mathbf{K}}$ is a regularisation matrix. Indeed, by adding two unknowns per node, a regularisation term is needed. But due to a formulation in the world reference system, the physical displacement is the unknown of the problem. Thus, it is easy to add a mechanical operator (such as $\mathbf{K V}=\mathbf{F}$ for an elastic deformation) in order to regularise the functional. But here, for our study, it is not possible to have the external forces. Thus, the stiffness matrix $\mathbf{K}$ is replaced by $\overline{\mathbf{K}}$.

The latter regularisation matrix is defined by $\overline{\mathbf{K}}=P_{k} \mathbf{K}$ with $P_{k}$ a diagonal matrix (7). The latter could be boolean, but in this study, $P_{k}$ is used to weight some dofs (eg to weigh the influence of rotations with respect to the membrane 
behaviour):

$$
\begin{cases}P_{k}^{i j}=0 & \forall j \neq i \\ P_{k}^{i i}=0 & \text { for all } \text { dofs on the non-free edges } \\ P_{k}^{i i}=0 & \text { for the membrane dofs related to } V_{x} \text { and } V_{y} \\ P_{k}^{i i}=1 & \text { for the dofs related to } V_{z} \\ P_{k}^{i i}=\frac{1}{h^{2}} & \text { for the dofs related to } \theta_{x} \text { and } \theta_{y}(h \text { being the element size) }\end{cases}
$$

Thus, $\overline{\mathbf{K}}$ is a matrix containing each $d o f$ except those supported by the nodes that are concerned with Dirichlet boundary conditions [20].

At iteration $k$, the problem reads:

$$
\underbrace{\left[\frac{1-\lambda_{m}}{\alpha_{v}} \Pi^{T} \mathbf{M}_{\text {stereo }}^{k} \Pi+\frac{\lambda_{m}}{\alpha_{m}} \overline{\mathbf{K}}^{T} \overline{\mathbf{K}}\right]}_{\mathbf{M}_{\overline{\mathbf{K}}}} \delta \mathbf{q}=\frac{1-\lambda_{m}}{\alpha_{v}} \Pi^{T} \mathbf{b}_{\text {stereo }}^{k}-\frac{\lambda_{m}}{\alpha_{m}} \overline{\mathbf{K}}^{T} \overline{\mathbf{K}} \mathbf{q}_{0}
$$

Remark. Replacing $\mathbf{K}$ by $\overline{\mathbf{K}}$ means no regularisation on the degrees of freedom of the non-free edges. Thus the behaviour of the nodes of these edges is only towed by the vision term. But in this study, a plate model is used, which means that the unknowns are 3 displacements and 2 rotations at the mid-plate surface. There is an additional difficulty because each dof on the non-free edges implies a singularity in $\mathbf{M}_{\overline{\mathbf{K}}}$. On the one hand, if these dofs are not taken into account, the problem cannot be solved. On the other hand, if they are removed from $\mathbf{M}_{\overline{\mathbf{K}}}$, it means that the associated nodes are clamped. Thus, there is a difficulty that will be solved in the next section 3.2 , especially for the rotations. Indeed, the displacement dofs can be considered in $\mathbf{M}_{\text {stereo }}^{k}$ but not the rotation dofs.

\subsection{Mechanical and Tikhonov Regularisation}

To overcome the absence of mechanical regularisation on the non-free edges (particularly for rotations of these nodes in question), it is possible to add a Tikhonov 
regularisation term (minimisation of a Laplacian term):

$$
\begin{aligned}
\mathbf{V}^{\star}=\underset{\mathbf{V}}{\arg \min } & \frac{1-\lambda_{m}-\lambda_{t}}{\alpha_{v}} \sum_{c} \int_{\Omega}\left[f_{c}\left(\mathbf{P}_{c}\left(\mathbf{X}, \mathbf{p}_{c}\right)\right)-g_{c}\left(\mathbf{P}_{c}\left(\mathbf{X}+\Pi \mathbf{V}(\mathbf{X}), \mathbf{p}_{c}\right)\right)\right]^{2} d \mathbf{X} \\
& +\frac{\lambda_{m}}{\alpha_{m}}\|\overline{\mathbf{K}} \mathbf{V}\|_{2}^{2}+\frac{\lambda_{t}}{\alpha_{t}}\|\Delta \mathbf{V}\|_{2}^{2}
\end{aligned}
$$

which gives:

$$
\begin{aligned}
& {\left[\frac{1-\lambda_{m}-\lambda_{t}}{\alpha_{v}} \Pi^{T} \mathbf{M}_{\text {stereo }}^{k} \Pi+\frac{\lambda_{m}}{\alpha_{m}} \overline{\mathbf{K}}^{T} \overline{\mathbf{K}}+\frac{\lambda_{t}}{\alpha_{t}} \mathbf{T}^{T} \mathbf{T}\right] \delta \mathbf{q}} \\
& =\frac{1-\lambda_{m}-\lambda_{t}}{\alpha_{v}} \Pi^{T} \mathbf{b}_{\text {stereo }}^{k}-\frac{\lambda_{m}}{\alpha_{m}} \overline{\mathbf{K}}^{T} \overline{\mathbf{K}} \mathbf{q}_{0}-\frac{\lambda_{t}}{\alpha_{t}} \mathbf{T}^{T} \mathbf{T} \mathbf{q}_{0}
\end{aligned}
$$

where $\mathbf{T}$ is the Tikhonov matrix corresponding to the Laplacian term, $\alpha_{t}$ is a weighting coefficient and $\lambda_{t}$ is a second dimensionless coefficient for this regularisation term set between 0 and 1 with respect to $\lambda_{m}$ (see Sect.4.1.3). Concretely, each node excluded from $\mathbf{K}$ will be associated to a zero eigenvalue. Adding the Laplacian term amounts to impose that those nodes adopt a similar behaviour than their neighbour.

Remark. The normalisation coefficients $\alpha_{v}$ and $\alpha_{m}$ are computed with the initial guess of the displacement $\mathbf{U}^{0}$. For $\alpha_{t}$, the goal is to have the smallest Laplacian term, thus, it has been chosen to set it at 1 . The coefficients are :

$$
\left\{\begin{aligned}
\alpha_{v} & =\mathbf{U}^{0^{T}} \mathbf{M}_{\text {stereo }}^{k} \mathbf{U}^{0} \\
\alpha_{m} & =\mathbf{U}^{0^{T}} \overline{\mathbf{K}}{ }^{T} \overline{\mathbf{K}} \mathbf{U}^{0} \\
\alpha_{t} & =1
\end{aligned}\right.
$$

\section{Application}

4.1 Synthetic test case

In order to quantify the measurement error with the described regularisation method, the goal here is to create some synthetic images for a plate test case. A pair of real images taken during a test is used (Fig. 
Both cameras being calibrated, the two projectors $\mathbf{P}_{c}$ are known. On the initial plate, for each camera $c$, the $3 \mathrm{D}$ reference points of the plate $\mathbf{X}_{r}=(X, Y, 0)^{T}$ are also known. It therefore suffices to determine the deformed points (according to a plate model) corresponding to each reference point to create a new image. According to the small strain and small displacement assumption, each point originally located at position $\mathbf{X}_{r}=(X, Y, 0)^{T}$ will be deformed to the point $\mathbf{X}_{d}=(X, Y, p(X, Y))^{T}$ with $p$ a function giving the out-of-plane position of a point according to a chosen plate model. Thus, for each integer coordinated $\mathbf{x}$ (each pixel of the new image), two unknowns $(X, Y)$ are solution of a system of two equations that can be solved by a Newton algorithm (Fig. 7(a)p:

$$
\mathbf{P}_{c}\left((X, Y, p(X, Y))^{T}\right)-\mathbf{x}=0
$$

Then, it is known that this deformed point was originally located at position $\mathbf{X}_{r}=(X, Y, 0)^{T}$ (Fig. 7(b). It is then possible to create a synthetic deformed image by interpolating the gray level of the reference image $f_{c}$ at the pixel $\mathbf{x}$ by projecting the reference $3 \mathrm{D}$ point $\mathbf{P}_{c}\left(\mathbf{X}_{r}\right)$ (Fig. $7(\mathrm{c})$.

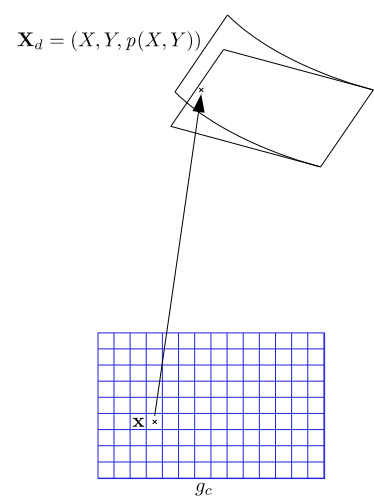

(a)
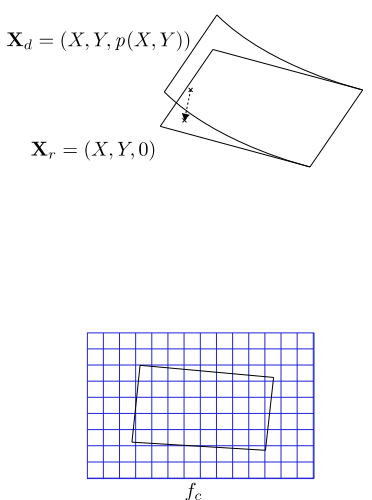

(b)

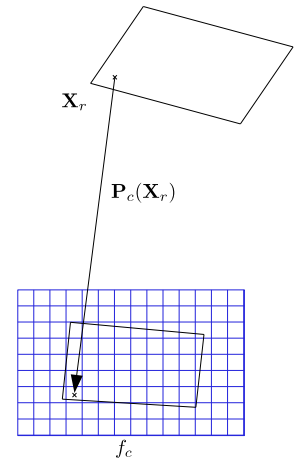

(c)

Fig. 7 Creation of a deformed synthetic image with a plate model $p$. (a) search for the $3 \mathrm{D}$ deformed point $\mathbf{X}_{d}$ according to a plate model $p$ which correspond to the pixel $\mathbf{x}$. (b) projection of this deformed point to the initial shape in order to find the reference $3 \mathrm{D}$ point $\mathbf{X}_{r}$. (c) interpolation of the reference image in order to associate a gray level with the pixel $\mathbf{x}$ of the created deformed image 
With this method, a pair of synthetic deformed image is created for a bending plate study. It is then possible to compute the measurement error by comparing the measure to the prescribed model $p$.

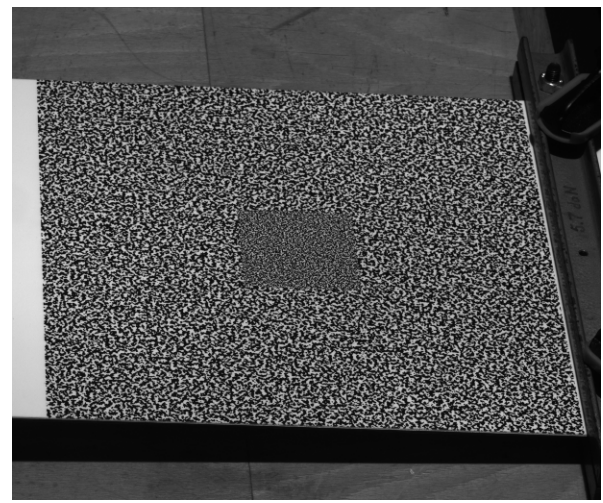

(a)

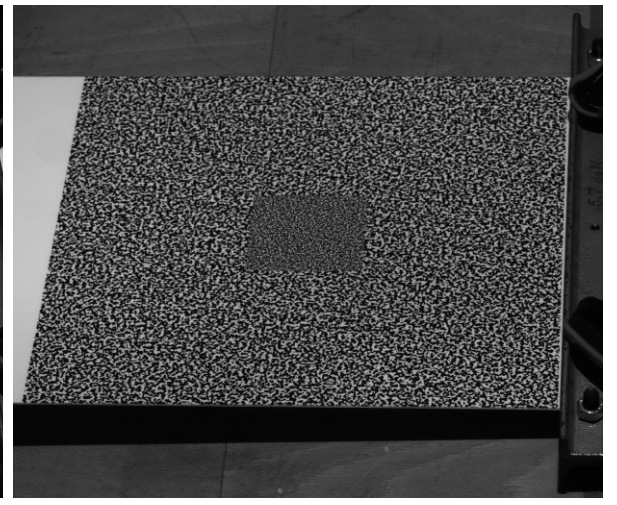

(b)

Fig. 8 Real images of a plate in bending. Reference images of left (a) and right (b) camera used to create synthetic deformed images with a plate model $p$. The plate size is $26.5 \times 26.5 \mathrm{~cm}^{2}$

\subsubsection{Mesh size influence}

The idea here is to calculate the measurement error according to the mean size of elements of an FE mesh. Both FE-SDIC and R-FE-SDIC (regularised, here with a plate model) are used. Fig. 9 presents the standard deviation of the error with both method when measuring the out-of-plane displacement $V_{z}$ and the rotation $\theta_{y}$. For the latter, with an FE-SDIC method, only the 3D displacement of the upper skin $\mathbf{U}$ is measured and the rotations are calculated using the derivative of the out-of-plane displacement $U_{z}=V_{z}$.

It can be seen in Fig. 9(a) that when the mesh size decreases, the measurement error also decreases due to the "FE error" until a mean element size of approximately $10 \mathrm{~mm}$. Then, because of a lack of pixels in the projected elements, the error increases, which corresponds to the "DIC error". In contrast, in blue, it can be noted that thanks to the mechanical regularisation, the error does not increase. 


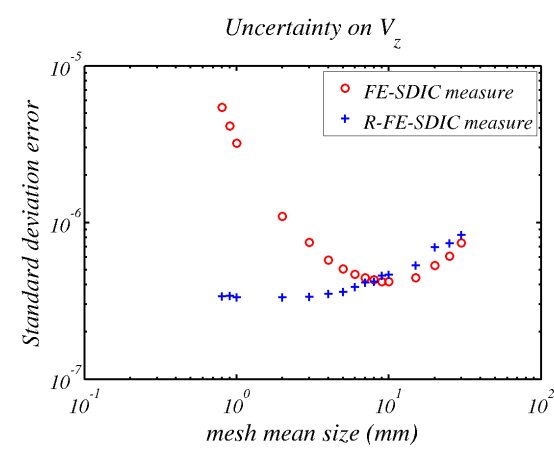

(a)

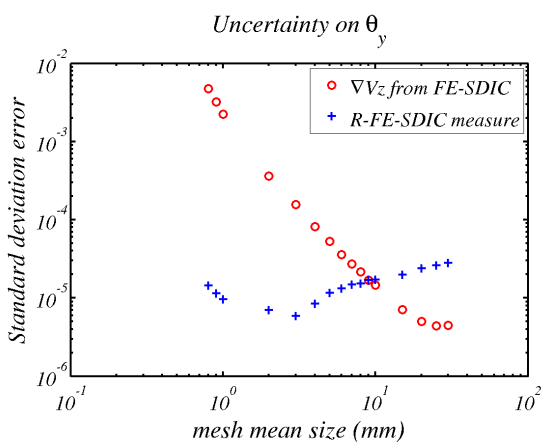

(b)

Fig. 9 Standard deviation of the error when measuring the out-of-plane displacement $V_{z}$ (a) and the rotation $\theta_{y}$ (b) for different sizes of mesh. This is computed with both methods: FE-SDIC (red) and R-FE-SDIC regularised measure with a plate model (blue)

Furthermore, in Fig. 9(b), it is shown that it is possible to directly measure the rotations regardless the mean element size. Furthermore, as expected, the derivative of a measured field (in red) is necessarily more noisy than a measured one (in blue).

\subsubsection{Noise robustness}

It is also possible to study the behaviour of measurement uncertainties when adding different level of noise when creating the deformed synthetic images $c f$. Fig. 10 For that, we added a zero-mean white Gaussian noise with a increasing standard deviation ranging from 1 to 8 graylevels. Thanks to the mechanical regularisation, the measurement is much more robust with noise.

Remarks:

- In this study, the image noise have been estimated to be less than 3 grey-levels (Allied Vision Pike FireWire 5 Megapixels).

- No filtering algorithm has been used in this work. The only regularisation is based on the use of a mechanical operator. 


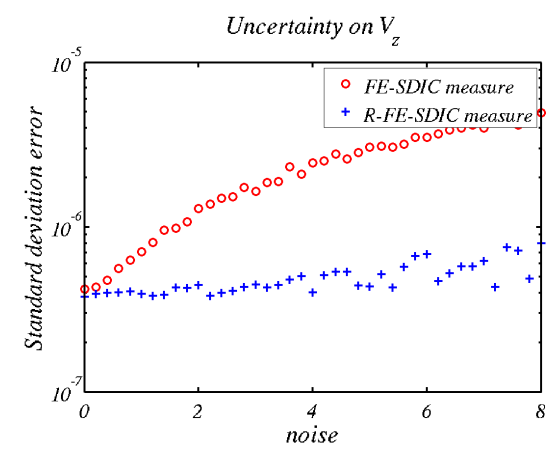

(a)

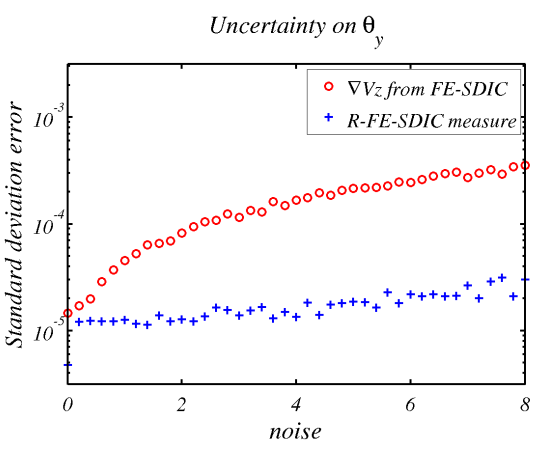

(b)

Fig. 10 Standard deviation of the error when measuring the out-of-plane displacement $V_{z}$ (a) and the rotation $\theta_{y}$ (b) as a function of the standard deviation of the noise. This is computed with both methods: FE-SDIC (red) and R-FE-SDIC regularised measure with a plate model (blue)

\subsubsection{Regularisation coefficients}

Obviously, the result from such a measurement depends on the used parameters $\lambda_{m}$ and $\lambda_{t}$. They are the cut-off wavelength of low-pass filters [10,8, 21]. Usually, this kind of parameters are set between 0 and 1 (eq. (9p). Thus, the three terms of (8) are normalized with $\alpha_{v}, \alpha_{m}$ and $\alpha_{t}$.

$$
\left\{\begin{array}{ccc}
0 \leq \underbrace{1-\lambda_{m}-\lambda_{t}}_{\lambda_{\text {vision }}} & \leq 1 \\
0 \leq & \lambda_{m} & \leq 1 \\
0 \leq & \lambda_{t} & \leq 1
\end{array}\right.
$$

Thus, different values of $\lambda_{m}$ can be used in order to study the measurement error. Without mechanical regularisation, a displacement can be observed but not a rotation. Thus, the rotation measurement with a low value of $\lambda_{m}$ corresponding to a low mechanical regularisation should not be less accurate than with a greater regularisation coefficient. But, as it can be seen in Figs.11(a) and (c), it is hard to find the optimal parameter, especially when looking to the rotation. The simplest way to find it for such an approach, is to draw a L-curve [12,7] which represent 
both DIC and Mechanical residual. The optimal parameter will naturally be at the corner of the L-curve $c f$. Fig. $11(\mathrm{e})$

On the other hand, the parameter $\lambda_{t}$ aims to take into account the degrees of freedom with a Dirichlet condition. Thus, the only condition seems to have a non-zero parameter: $\lambda_{t} \neq 0$. But it can be seen in Figs. 11(b) (d) and (f) that this parameter does have an impact on the measurement. Indeed, the weight of this coefficient has an influence on the boundary. And since boundary displacement uncertainty has a tendency to spread in the mechanical regularised region, it has an effect on the solution [8].

\subsection{Real test case}

The same measurement is now carried out with real deformed images. As expected, the measurement of the rotation by R-FE-SDIC (mechanically regularised FE-Stereo-DIC with a plate theory) is more accurate than without mechanical regularisation, because the latter is the numerical derivative of a measured field cf. Fig. 12 .

The main advantage of a Stereo method based on the world reference system is that it is possible to use a real mesh. Indeed, in a classical FE-SDIC method, the mesh has to be adapted to the measurement. Here, the goal is to adapt the measurement in order to realize a real dialogue between experiment and simulation. The mesh chosen for a simulation can also be used for a measurement. Thus, for example, the mesh can be refined in the center for a potential default $c f$. Fig. 13 . In this figure, the shape measurement step is compared to the one computed by Vic- $3 \mathrm{D}^{\mathrm{TM}}$. It can be seen that the point cloud resulting from Vic- $3 \mathrm{D}^{\mathrm{TM}}$ is close to the mesh except at the boundary where Vic- $3 \mathrm{D}^{\mathrm{TM}}$ shape is not available.

With this kind of mesh, even the displacement field computed without regularisation would not be correct because of the mesh refinement at the center $c f$. Fig. 14 . 


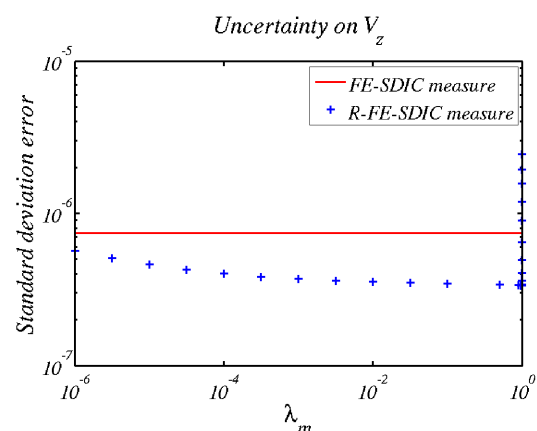

(a)

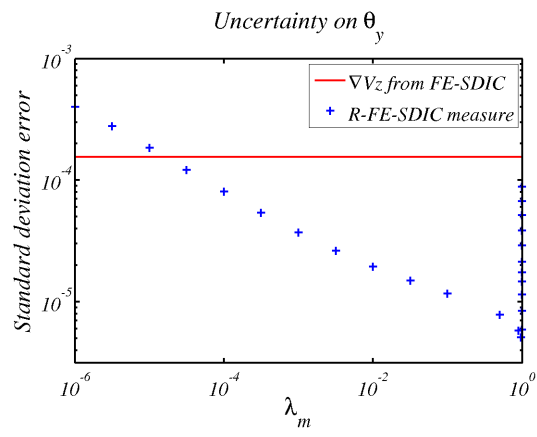

(c)

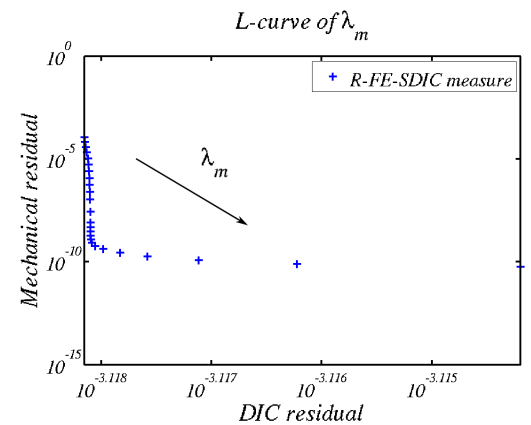

(e)

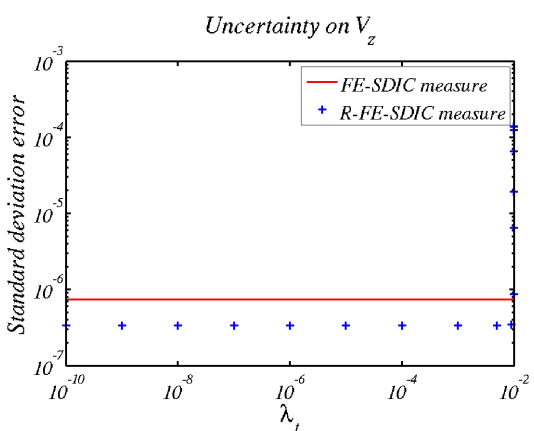

(b)

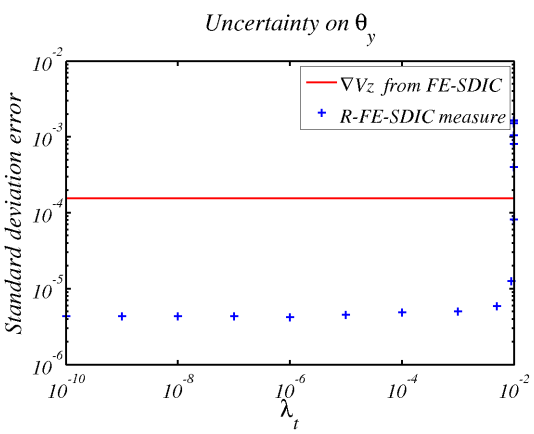

(d)

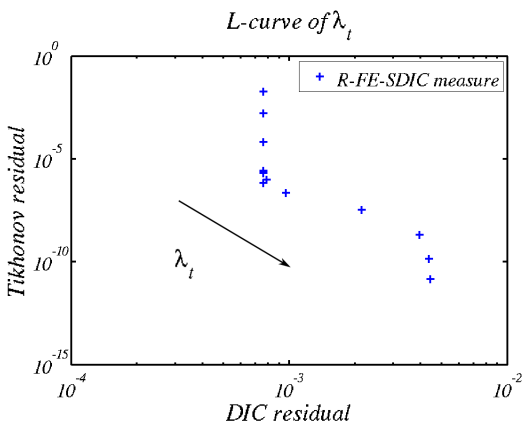

(f)

Fig. 11 Schemes for studying the influence of $\lambda_{m}$ (left) and $\lambda_{t}$ (right). Standard deviation of the error when measuring the out-of-plane displacement $V_{z}(\mathrm{a}, \mathrm{b})$ and the rotation $\theta_{y}(\mathrm{c}, \mathrm{d})$ for different values of $\lambda_{m}$ and $\lambda_{t}$. This is computed with R-FE-SDIC regularised measure with a plate model (blue) and the value corresponding to the FE-SDIC method is plotted (red) for a comparison. L-curve (e, f) of both parameter in order to minimise both DIC and Mechanical residual 


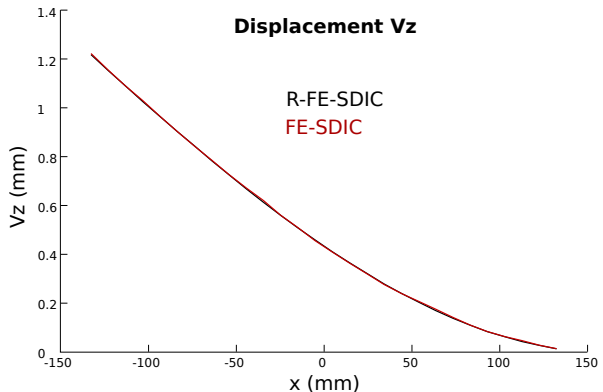

(a)

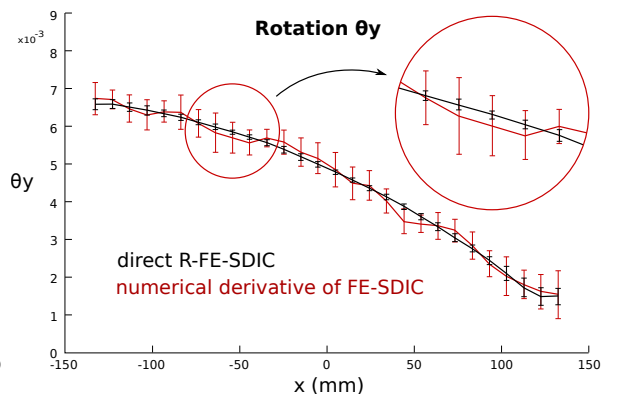

(b)

Fig. 12 Comparison of the measurement of the displacement $V_{z}$ (a) or the rotation $\theta_{y}$ (b) with different approaches : FE-SDIC (red) or R-FE-SDIC (black)
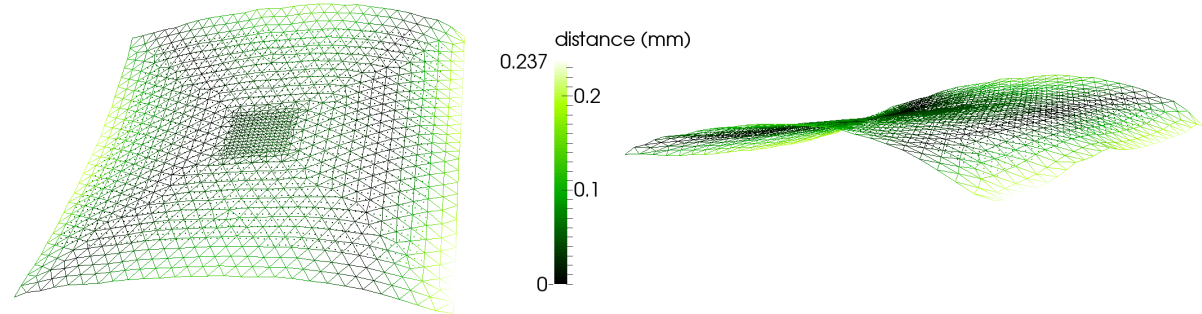

Fig. 13 Shape of a large plate measured by SDIC during an actual experiment. A suitable mesh is devised to perform a better resolved FE-SDIC measurement around a potential defect located in the center of the plate. The FE-SDIC shape measurement (mesh) is compared to a classical SDIC shape measurement (Vic-3D ${ }^{\mathrm{TM}}$ : point cloud)

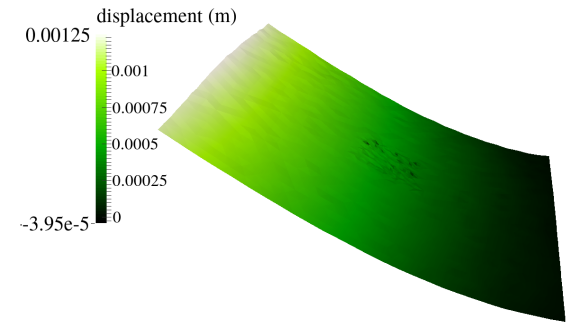

(a)

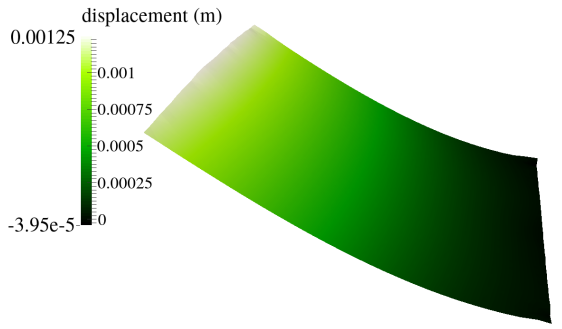

(b)

Fig. 14 Comparison of the out-of-plane displacement fields $V_{z}$ measured by FE-SDIC without regularisation (a), or with regularisation R-FE-SDIC (b) 


\section{Conclusion}

A full stereovision framework for measuring the 3D shape and displacements using a Finite Element mesh was developed. The formulation incorporates a gray-level based calibration step extended to non-linear camera models. It was shown that, in opposition to stereovision using NURBS functions [1, the shape measurement is very ill-posed and requires additional regularisation. We used for that a type of isometric constraint. Thanks to this formulation in the world coordinate system, the same FE mesh can be shared between both numerical simulation and measurement. It is thus a good tool to perform quantitative comparisons between non-planar experiments and corresponding simulations that are most often based on Finite Elements [26,27]. In addition, a technique was proposed to build the minimal number of integration points in such a context.

As an application, the method was used for the measurement of the displacement and rotation fields of a plate in bending, using a mechanical regularisation based on the elastic stiffness operator associated to the FE mesh. In addition, stereo synthetic test cases are developed and analysed in order to exemplify the efficiency of the proposed method.

Acknowledgements This work was funded by the French "Agence Nationale de la Recherche" under the grant ANR-12-RMNP-0001 (VERTEX project).

\section{References}

1. Beaubier, B., Dufour, J.E., Hild, F., Roux, S., Lavernhe, S., Lavernhe-Taillard, K.: CADbased calibration and shape measurement with stereoDIC: Principle and application on test and industrial parts. Experimental Mechanics 54(3), 329-341 (2014). DOI 10.1007/ s11340-013-9794-6.

2. Besnard, G., Hild, F., Roux, S.: "Finite-Element" Displacement Fields Analysis from Digital Images: Application to Portevin-Le Châtelier Bands. Experimental Mechanics 46(6), 789-803 (2006). DOI 10.1007/s11340-006-9824-8. 
3. Dufour, J.E., Beaubier, B., Hild, F., Roux, S.: CAD-based Displacement Measurements with Stereo-DIC: Principle and First Validations. Experimental Mechanics (2015). DOI 10.1007/s11340-015-0065-6.

4. Dufour, J.E., Hild, F., Roux, S.: Shape, displacement and mechanical properties from isogeometric multiview stereocorrelation. The Journal of Strain Analysis for Engineering Design (2015). DOI 10.1177/0309324715592530.

5. Garcia, D., Orteu, J.J.: 3d deformation measurement using stereo-correlation applied to experimental mechanics. In: Proceedings of the 10th FIG International Symposium Deformation Measurements, pp. 19-22 (2001).

6. Horn, B.K.P., Schunck, B.G.: Determining optical flow. Artificial Intelligence 17(1-3), 185-203 (1981). DOI 10.1016/0004-3702(81)90024-2

7. Lawson, C., Hanson, R.: Solving Least Squares Problems. Society for Industrial and Applied Mathematics (1995). DOI 10.1137/1.9781611971217.

8. Leclerc, H., Périé, J.N., Hild, F., Roux, S.: Digital volume correlation: what are the limits to the spatial resolution? Mechanics \& Industry 13(06), 361-371 (2012).

9. Leclerc, H., Périé, J.N., Roux, S., Hild, F.: Integrated Digital Image Correlation for the Identification of Mechanical Properties. In: A. Gagalowicz, W. Philips (eds.) Computer Vision/Computer Graphics CollaborationTechniques, no. 5496 in Lecture Notes in Computer Science, pp. 161-171. Springer Berlin Heidelberg (2009).

10. Leclerc, H., Périé, J.N., Roux, S., Hild, F.: Voxel-Scale Digital Volume Correlation. Experimental Mechanics 51(4), 479-490 (2011). DOI 10.1007/s11340-010-9407-6.

11. Lucas, B.D., Kanade, T.: An iterative image registration technique with an application to stereo vision. In: Proceedings of Imaging Understanding Workshop, pp. 121-130 (1981).

12. Miller, K.: Least squares methods for ill-posed problems with a prescribed bound. SIAM Journal on Mathematical Analysis 1(1), 52-74 (1970). DOI 10.1137/0501006.

13. Passieux, J.C.: Quelques outils numériques pour la simulation et la mesure en mécanique des structures. Habilitation à diriger des recherches de l'Université de Toulouse 122p. (2015). http://hal.archives-ouvertes.fr/tel-01370556.

14. Passieux, J.C., Bugarin, F., David, C., Périé, J.N., Robert, L.: Multiscale Displacement Field Measurement Using Digital Image Correlation: Application to the Identification of Elastic Properties. Experimental Mechanics 55(1), 121-137 (2015). DOI 10.1007/ s11340-014-9872-4.

15. Passieux, J.C., Périé, J.N., Salaün, M.: A dual domain decomposition method for finite element digital image correlation. International Journal for Numerical Methods in Engineering 102(10), 1670-1682 (2015). DOI 10.1002/nme.4868. 
16. Passieux, J.C., Réthoré, J., Gravouil, A., Baietto, M.C.: Local/global non-intrusive crack propagation simulation using a multigrid X-FEM solver. Computational Mechanics 52(6), 1381-1393 (2013). DOI 10.1007/s00466-013-0882-3.

17. Pierré, J.E., Passieux, J.C., Périé, J.N., Bugarin, F., Robert, L.: Unstructured finite element-based digital image correlation with enhanced management of quadrature and lens distortions. Optics and Lasers in Engineering 77, 44-53 (2016). DOI 10.1016/j. optlaseng.2015.07.008.

18. Roux, S., Hild, F.: Stress intensity factor measurements from digital image correlation: post-processing and integrated approaches. International Journal of Fracture 140(1-4), 141-157 (2006). DOI 10.1007/s10704-006-6631-2.

19. Roux, S., Réthoré, J., Hild, F.: Digital image correlation and fracture: an advanced technique for estimating stress intensity factors of $2 \mathrm{~d}$ and $3 \mathrm{~d}$ cracks. Journal of Physics D: Applied Physics 42(21), 214,004 (2009). DOI 10.1088/0022-3727/42/21/214004.

20. Réthoré, J.: A fully integrated noise robust strategy for the identification of constitutive laws from digital images. International Journal for Numerical Methods in Engineering 84(6), 631-660 (2010). DOI 10.1002/nme.2908.

21. Réthoré, J.: Automatic crack tip detection and stress intensity factors estimation of curved cracks from digital images: AUTOMATIC CRACK TIP DETECTION AND SIF ESTIMATION OF CURVED CRACKS. International Journal for Numerical Methods in Engineering 103(7), 516-534 (2015). DOI 10.1002/nme.4905.

22. Réthoré, J., Muhibullah, Elguedj, T., Coret, M., Chaudet, P., Combescure, A.: Robust identification of elasto-plastic constitutive law parameters from digital images using $3 \mathrm{~d}$ kinematics. International Journal of Solids and Structures 50(1), 73-85 (2013). DOI 10.1016/j.ijsolstr.2012.09.002.

23. Sun, Y., Pang, J.H.L., Wong, C.K., Su, F.: Finite element formulation for a digital image correlation method. Applied Optics 44(34), 7357-7363 (2005). DOI 10.1364/AO.44. 007357.

24. Sutton, M.A., Wolters, W.J., Peters, W.H., Ranson, W.F., McNeill, S.R.: Determination of displacements using an improved digital correlation method. Image and Vision Computing 1(3), 133-139 (1983). DOI 10.1016/0262-8856(83)90064-1.

25. Sutton, M.A., Yan, J.H., Tiwari, V., Schreier, H.W., Orteu, J.J.: The effect of out-ofplane motion on $2 \mathrm{~d}$ and $3 \mathrm{~d}$ digital image correlation measurements. Optics and Lasers in Engineering 46(10), 746-757 (2008). DOI 10.1016/j.optlaseng.2008.05.005.

26. Sztefek, P., Olsson, R.: Tensile stiffness distribution in impacted composite laminates determined by an inverse method. Composites Part A: Applied Science and Manufacturing 39(8), 1282-1293 (2008). DOI 10.1016/j.compositesa.2007.10.005. 
27. Sztefek, P., Olsson, R.: Nonlinear compressive stiffness in impacted composite laminates determined by an inverse method. Composites Part A: Applied Science and Manufacturing 40(3), 260-272 (2009). DOI 10.1016/j.compositesa.2008.12.002.

28. Triggs, B., McLauchlan, P.F., Hartley, R.I., Fitzgibbon, A.W.: Bundle adjustment - a modern synthesis. In: Vision algorithms: theory and practice, pp. 298-372. Springer (2000). 